OPEN ACCESS

Edited by:

Gong Zhang,

Jinan University, China

Reviewed by:

Yi Zhang,

Zunyi Medical University, China

Bing Bai,

Nanjing Drum Tower Hospital, China

*Correspondence:

Na Shen

shenna@tjh.tjmu.edu.cn

Specialty section: This article was submitted to

Cancer Genetics,

a section of the journal

Frontiers in Oncology

Received: 09 December 2020 Accepted: 04 May 2021

Published: 25 May 2021

Citation:

Wang P, Li Y, Li L, Zhong $R$ and

Shen N (2021) MBOAT7-TMC4 rs641738 is Not Associated With the Risk of Hepatocellular Carcinoma or Persistent Hepatitis B Infection.

Front. Oncol. 11:639438.

doi: $10.3389 /$ fonc. 2021.639438

\section{MBOAT7-TMC4 rs641738 Is Not Associated With the Risk of Hepatocellular Carcinoma or Persistent Hepatitis B Infection}

\author{
Peng Wang ${ }^{1}$, Ying $L i^{2}$, Lu $L i^{3}$, Rong Zhong ${ }^{4}$ and Na Shen ${ }^{2 *}$ \\ 1 Institute and Department of Infectious Disease, Tongji Hospital, Tongji Medical College, Huazhong University of Science and \\ Technology, Wuhan, China, ${ }^{2}$ Department of Laboratory Medicine, Tongji Hospital, Tongji Medical College, Huazhong \\ University of Science and Technology, Wuhan, China, ${ }^{3}$ Research Center for Translational Medicine, Shantou University \\ Medical College, Shantou, China, ${ }^{4}$ Department of Epidemiology and Biostatistics, MOE Key Laboratory of Environment \& \\ Health, School of Public Health, Tongji Medical College, Huazhong University of Science and Technology, Wuhan, China
}

Objective: A hot genetic variant, rs641738 within the membrane-bound Oacyltransferase domain containing 7(MBOAT7) and transmembrane channel-like 4 (TMC4), was recently reported to be associated with several liver diseases. However, the results remain controversial. Therefore, this study aimed to explore the role of MBOAT7-TMC4 rs641738 in the risk of hepatocellular carcinoma (HCC) and persistent hepatitis $B$ virus (HBV) infection.

Methods: We first conducted a case-control study that included 779 HCC cases and 1412 cancer-free controls. Controls consisted of 678 persistent HBV carriers and 734 spontaneously recovered subjects. The gene variant rs641738 was genotyped using the MassARRAY platform. The results were analyzed in five genetic models using multivariate logistic regression analyses. Next, we performed a systematic review and meta-analysis to further explore the role of this variant in HCC risk.

Results: The results suggested no association between MBOAT7-TMC4 rs641738 and HCC risk in most genetic models (all $P>0.05$ ). Although a marginally significant association was observed in $\Pi$ vs. CC $(P=0.037)$ and the recessive models $(P=$ 0.044). The meta-analysis of $2135 \mathrm{HCC}$ cases and 4388 controls supported that this variant was not related to HCC risk, even in the $\Pi \mathrm{vs}$. CC and recessive models. We also determined that this variant did not influence persistent HBV infection.

Conclusion: Our work highlights that MBOAT7-TMC4 rs641738 is not associated with the risk of HCC or persistent HBV infection. This study provides some clues to identify the "truth" of potential disease-related genetic factors in the post-genome era.

Keywords: hepatocellular carcinoma, persistent HBV infection, rs641738, susceptibility, MBOAT7 gene, casecontrol study 


\section{INTRODUCTION}

Hepatocellular carcinoma (HCC) is a common rapidly progressing cancer, with high mortality worldwide, especially in China (1). As the fourth most common cancer in China, 466,100 new cases were estimated in 2015 (2). Environmental factors such as hepatitis B virus (HBV) infection have been confirmed to be crucial in the pathogenesis of HCC (3). Increasing evidence has revealed that genetic factors also play an important role in the development of HCC (4).

HCC is a complicated disease with high genetic heterogeneity. Candidate gene studies and genome-wide association studies (GWASs) have identified hundreds of genes and loci associated with HCC risk. How to identify the "truth" of these genetic factors is becoming an urgent issue in the post-genome era. In 2015, a GWAS study reported that a genetic variant near the membrane-bound O-acyltransferase domain containing 7 (MBOAT7) gene, rs641738, C>T, increased the risk of alcoholrelated cirrhosis (5). It is also reported to be located in the transmembrane channel-like 4 (TMC4) gene, which is not abundantly expressed in the human liver (6). The rs641738 variant is mapped to 500-bp downstream of MBOAT7 and is located in exon 1 of the TMC4 gene. Helsley et al. first reported that genetic deletion of Tmc4 in mice may not lead to hepatic steatosis, but the loss of function of its neighboring gene Mboat7 could result in liver disease progression in mice (7). Therefore, the rs641738 variant is often referred to as the MBOAT7 or MBOAT7-TMC4 variant. In 2016, Thabet et al. demonstrated that MBOAT7-TMC4 rs641738 could be a risk factor for hepatic inflammation and liver fibrosis (8). Subsequent studies further investigated the association between this variant and HCC risk, but led to conflicting conclusions (9-11).

Here, we first conducted a case-control study including 779 HCC cases and 1,412 cancer-free controls, aiming to explore the effects of MBOAT7-TMC4 rs641738 on HCC risk in a Chinese population. We then performed a systematic review and metaanalysis to further explore the role of this variant and validate our results.

\section{MATERIALS AND METHODS}

\section{Study Subjects}

This case-control study included 779 HCC cases and 1,412 cancer-free controls. The cases were pathologically confirmed and enrolled between January 2014 and June 2016 at Tongji

\footnotetext{
Abbreviations: $M B O A T 7$, membrane bound O-acyltransferase domain containing 7; TMC4, transmembrane channel-like 4; HCC, hepatocellular carcinoma; HBV, hepatitis B virus; GWASs, genome-wide association studies; HUST, Huazhong University of Science and Technology; HBsAg, hepatitis B surface antigen; $\mathrm{HBcAb}$, hepatitis $\mathrm{B}$ core antibody; anti-HCV, antibody against hepatitis $\mathrm{C}$ virus; HBsAb, hepatitis B surface antibody; ELISA, Enzyme-linkedimmunosorbent assay; HWE, Hardy-Weinberg equilibrium; ANOVA, analysis of variance; OR, odds ratio; CI, confidence interval; PRISMA, Preferred Reporting Items for Systematic Review and Meta-analyses; NOS, Newcastle-Ottawa scale; vs., versus; NAFLD, nonalcoholic fatty liver disease; NASH, nonalcoholic steatohepatitis; SD, standard deviation.
}

Hospital of Huazhong University of Science and Technology (HUST), central China. The controls are consisted of 678 persistent HBV carriers and 734 spontaneously recovered subjects, who were recruited from a health screening in the same hospital during the same period as the cases were included. Persistent HBV carriers were people who were positive for both of the hepatitis B surface antigen ( $\mathrm{HBs} A g$ ) and hepatitis B core antibody ( $\mathrm{HBcAb})$, but negative for antibody against hepatitis $\mathrm{C}$ virus (anti-HCV). Spontaneously recovered subjects were people who were negative for both of $\mathrm{HBsAg}$ and anti-HCV, but positive for both of $\mathrm{HBcAb}$ and hepatitis B surface antibody (HBsAb). All subjects were unrelated Han Chinese from Wuhan and the surrounding regions. Cases and controls were frequencymatched for sex and age ( \pm 5 years). At recruitment, a $2-\mathrm{ml}$ peripheral blood sample and a written informed consent were collected from each subject, and demographic information (i.e., sex, age, smoking and drinking status) were also obtained by questionnaire. The definitions of smoking and drinking status have been detailed previously $(12,13)$. Briefly, smokers were defined as subjects who smoked at least one cigarette a day and smoked for more than a year before the date of the interview; otherwise, they were defined as non-smokers. Drinkers were defined as subjects who drunk at least twice a week and drunk for more than a year before the date of the interview; otherwise, they were defined as non-drinkers. This study was approved by the institutional ethics committee of Tongji Hospital, Tongji Medical College of HUST.

\section{Serological Testing}

Enzyme-linked immunosorbent assay (ELISA) was used to detect serum HBsAg, HBsAb, HBcAb, and anti-HCV (IMX; Abbott Diagnostics, USA). There were three positive controls, two negative controls, and one blank control in each reaction plate. About $5 \%$ of the samples were randomly chosen for repetition, and results were $100 \%$ concordant.

\section{Genotyping}

Genomic DNA was extracted from leucocyte pellets of 2-ml peripheral blood by the coagulated blood DNA mini-extraction kit (DP6101, BioTeke Corporation, China). MBOAT7-TMC4 rs641738 was genotyped using the Sequenom MassARRAY iPLEX Platform (SEQUENOM, CA, USA). All assays were conducted using a 384-well plate with positive and negative controls for each plate, without information on the disease status of the samples. We randomly selected $5 \%$ of the samples as duplicate sets and got a $100 \%$ concordance rate. The average call rate of this variant was $99.7 \%$. A polar plot of rs641738 is shown in Supplementary Figure S1.

\section{Statistical Analysis}

A goodness-of-fit $\chi^{2}$ test was applied to assess the HardyWeinberg equilibrium (HWE) in controls. Differences between cases and controls were examined by independent $\mathrm{t}$-test, analysis of variance (ANOVA), or Pearson's $\chi^{2}$ test according to the category of variables. The risk of HCC or persistent HBV infection was estimated by odds ratio (OR) and $95 \%$ confidence interval (CI), using a multivariate logistic regression 
analysis after adjusting for age, sex, smoking, and drinking status. Statistical significance was defined as a two-tailed $P$ value $<0.05$. All the analyses above were performed using IBM SPSS Statistics (version 20.0; Chicago, IL, USA). We calculated the statistical power of this study using Power V3.0 (14). Our sample size was determined based on an OR of 1.30 and a power of 0.95 .

\section{Meta-analysis}

This meta-analysis was performed according to the guidelines of the Preferred Reporting Items for Systematic Reviews and Metaanalyses (PRISMA) (15).

\section{Literature Search, Study Selection, and Data Extraction}

A comprehensive literature search was conducted through PubMed, Embase, and Web of Science databases up to December 2020 without any restrictions. The search items included "MBOAT7," "TMC4," "rs641738," and "hepatocellular carcinoma." References from the identified publications were also reviewed to obtain potentially potential relevant studies.

The inclusion criteria were as follows: (1) a case-control or cohort study to evaluate the association between MBOAT7-TMC4 rs641738 and HCC risk; (2) providing ORs and 95\%CIs, or allele frequency and/or genotypes of this variant; and (3) the number of cases was more than 30 . A study was excluded if it met one of the following criteria: (1) review, meta-analysis, comment, or conference abstract; (2) insufficient data to estimate OR and 95\%CI; (3) a deviation from HWE in controls; and (4) studies with overlapping data. If a study contained overlapping data with another, we retained the study with a larger sample size.

Data were extracted from each included study as follows: first author, publication year, country, ethnicity, sex, age, sample size, genotype distribution, genotyping method, adjustment, and HWE information. Two authors independently assessed the studies as described above. Disagreements were resolved through discussion. The quality of each included study was evaluated through the Newcastle-Ottawa scale (NOS) (16). For case-control studies, the NOS has eight items, which are categorized into three dimensions: selection, comparability, and exposure. The score of NOS ranges from zero to nine and a study with score $\geq 7$ is often considered as high-quality. Quality evaluation was not an exclusion criterion for eligible studies (Supplementary Table S1).

\section{Statistical Analysis for Meta-analysis}

We used ORs and 95\%CIs to estimate the association between MBOAT7-TMC4 rs641738 and HCC risk. Multivariate-adjusted ORs and 95\%CIs were preferentially extracted if available. Otherwise, the unadjusted ORs and 95\%CIs were calculated instead. Between-study heterogeneity was examined using the Q test and $I^{2}$ statistic. If $P<0.10$ or $I^{2}>50 \%$, significant heterogeneity was considered, and a random-effects model was applied. Otherwise, the fixed-effects model was used. To evaluate the quality, we performed a sensitivity analysis and publication test $(17,18)$. Sensitivity analysis was carried out by re-estimating pooled ORs and 95\%CIs after excluding each eligible study in turn, aiming to assess the stability of the pooled results. The meta-analysis was conducted using Stata 12.0 software (College Station, TX, USA).

\section{RESULTS}

\section{Subject Characteristics}

A total of 779 HCC cases, 678 persistent HBV carriers and 734 spontaneously recovered subjects were included in this study. Demographic characteristics are summarized in Table 1. In these three groups, the proportion of males in the three groups were $70.6 \%, 68.7 \%$, and $68.1 \%$, respectively. The mean ages were $53.20 \pm 12.49,52.27 \pm 11.45$, and $52.28 \pm 12.89$ years, respectively. There was no significant difference among the three groups on sex $(P=0.551)$, age $(P=0.240)$, smoking $(P=$ $0.071)$, and drinking status $(P=0.091)$. Genotypes of MBOAT7TMC4 rs641738 in all groups did not deviate from HWE (HCC: $P=0.485$; persistent $\mathrm{HBV}$ carriers: $P=0.133$; spontaneously recovered subjects: $P=0.769$ ).

\section{Association of MBOAT7-TMC4 rs641738 with Risk of HCC and Persistent HBV Infection}

We performed four comparisons under the five genetic models, and the results are shown in Table 2. In the comparison of HCC

TABLE 1 | The characteristics of the included subjects.

\begin{tabular}{|c|c|c|c|c|c|}
\hline Variables & HCC, N (\%) & $\begin{array}{l}\text { Persistent HBV } \\
\text { carriers, N (\%) }\end{array}$ & $\begin{array}{c}\text { Spontaneously recovered } \\
\text { subjects, N (\%) }\end{array}$ & $\chi^{2} / F$ & $P$ \\
\hline Total & 779 & 678 & 734 & & \\
\hline Sex & & & & 1.191 & 0.551 \\
\hline Male & $550(70.6)$ & $466(68.7)$ & $500(68.1)$ & & \\
\hline Female & 229 (29.4) & 212 (31.3) & 234 (31.9) & & \\
\hline Age (mean $\pm \mathrm{SD})$ & $53.20 \pm 12.49$ & $52.27 \pm 11.45$ & $52.28 \pm 12.89$ & 1.428 & 0.240 \\
\hline Smoking status & & & & 5.280 & 0.071 \\
\hline Smokers & 301 (38.6) & $243(35.8)$ & 242 (33.0) & & \\
\hline Non-smokers & $478(61.4)$ & 435 (64.2) & $492(67.0)$ & & \\
\hline Drinking status & & & & 4.803 & 0.091 \\
\hline Drinkers & $270(34.7)$ & 265 (39.1) & 291 (39.6) & & \\
\hline Non-drinkers & 509 (65.3) & $413(60.9)$ & $443(60.4)$ & & \\
\hline
\end{tabular}

$S D$, standard deviation. 
TABLE 2 | The effects of MBOAT7-TMC4 rs641738 on persistent HBV infection and HCC.

\begin{tabular}{|c|c|c|c|c|c|c|c|}
\hline Genotypes & HCC, N (\%) & $\begin{array}{l}\text { Persistent HBV } \\
\text { carriers, N (\%) }\end{array}$ & $\begin{array}{l}\text { Spontaneously } \\
\text { recovered } \\
\text { subjects, N (\%) }\end{array}$ & $\begin{array}{l}\text { Adjusted OR } \\
(95 \% \mathrm{Cl}), P^{a}\end{array}$ & $\begin{array}{l}\text { Adjusted OR } \\
(95 \% \mathrm{Cl}), P^{b}\end{array}$ & $\begin{array}{c}\text { Adjusted OR } \\
(95 \% \mathrm{Cl}), P^{c}\end{array}$ & $\begin{array}{l}\text { Adjusted OR } \\
(95 \% \mathrm{Cl}), P^{d}\end{array}$ \\
\hline $\mathrm{CC}$ & $426(54.7)$ & $380(56.1)$ & $420(57.7)$ & Reference & Reference & Reference & Reference \\
\hline $\mathrm{CT}$ & $295(37.9)$ & 264 (39.0) & 264 (36.3) & $\begin{array}{c}1.05(0.87-1.27) \\
0.604\end{array}$ & $\begin{array}{c}1.00(0.82-1.24) \\
0.981\end{array}$ & $\begin{array}{c}1.10(0.89-1.37) \\
0.370\end{array}$ & $\begin{array}{c}1.11(0.89-1.38) \\
0.366\end{array}$ \\
\hline Tा & $58(7.4)$ & $33(4.9)$ & $44(6.0)$ & $\begin{array}{c}1.47(1.02-2.12) \\
0.037\end{array}$ & $\begin{array}{c}1.64(1.04-2.57) \\
0.033\end{array}$ & $\begin{array}{c}1.35(0.89-2.05) \\
0.164\end{array}$ & $\begin{array}{c}0.85(0.53-1.36) \\
0.491\end{array}$ \\
\hline Dominant model & & & & $\begin{array}{c}1.10(0.92-1.32) \\
0.280\end{array}$ & $\begin{array}{c}1.07 \text { (0.87-1.32) } \\
0.541\end{array}$ & $\begin{array}{c}1.14(0.93-1.40) \\
0.219\end{array}$ & $\begin{array}{c}1.07(0.87-1.32) \\
0.529\end{array}$ \\
\hline $\begin{array}{l}\text { Recessive } \\
\text { model }\end{array}$ & & & & $\begin{array}{c}1.44(1.01-2.06) \\
0.044\end{array}$ & $\begin{array}{c}1.64(1.05-2.55) \\
0.029\end{array}$ & $\begin{array}{c}1.29(0.86-1.95) \\
0.218\end{array}$ & $\begin{array}{c}0.81(0.51-1.29) \\
0.383\end{array}$ \\
\hline Additive model & & & & $\begin{array}{c}1.13(0.98-1.31) \\
0.094\end{array}$ & $\begin{array}{c}1.13(0.95-1.33) \\
0.172\end{array}$ & $\begin{array}{c}1.13(0.96-1.34) \\
0.138\end{array}$ & $\begin{array}{c}1.02(0.85-1.21), \\
0.851\end{array}$ \\
\hline Allelic model (T vs. & C) & & & $\begin{array}{c}1.13(0.98-1.30) \\
0.095\end{array}$ & $\begin{array}{c}1.12(0.95-1.33) \\
0.175\end{array}$ & $\begin{array}{c}1.14(0.96-1.34) \\
0.135\end{array}$ & $\begin{array}{c}1.02(0.86-1.21), \\
0.853\end{array}$ \\
\hline
\end{tabular}

${ }^{a}$ HCC vs. (persistent HBV carriers + spontaneously recovered subjects), adjusted for sex, age, smoking, and drinking status.

${ }^{b}$ HCC vs. persistent HBV carriers, adjusted for sex, age, smoking, and drinking status.

${ }^{c}$ HCC vs. spontaneously recovered subjects, adjusted for sex, age, smoking, and drinking status.

${ }^{d}$ Persistent HBV carriers vs. spontaneously recovered subjects, adjusted for sex, age, smoking, and drinking status.

cases versus ( $v s$.$) all controls, we found that after adjusting for$ sex, age, smoking, and drinking status, MBOAT7-TMC4 rs641738 did not confer any increased risk of HCC in the dominant, additive, or allelic models (all $P>0.05$ ). Although this variant seemed to be a risk factor for HCC in the two models, the results were marginally significant (TT vs. CC: $P=0.037$; recessive: $P=0.044)$. Similar results were also observed in a subsequent comparison of HCC vs. persistent HBV carriers. When we set spontaneously recovered subjects as controls, we observed that MBOAT7-TMC4 rs641738 was not associated with HCC risk (all $P>0.10$ ). To explore whether this variant influenced the risk of persistent $\mathrm{HBV}$ infection, we conducted a further comparison between persistent HBV carriers and spontaneously recovered subjects. The results suggested that

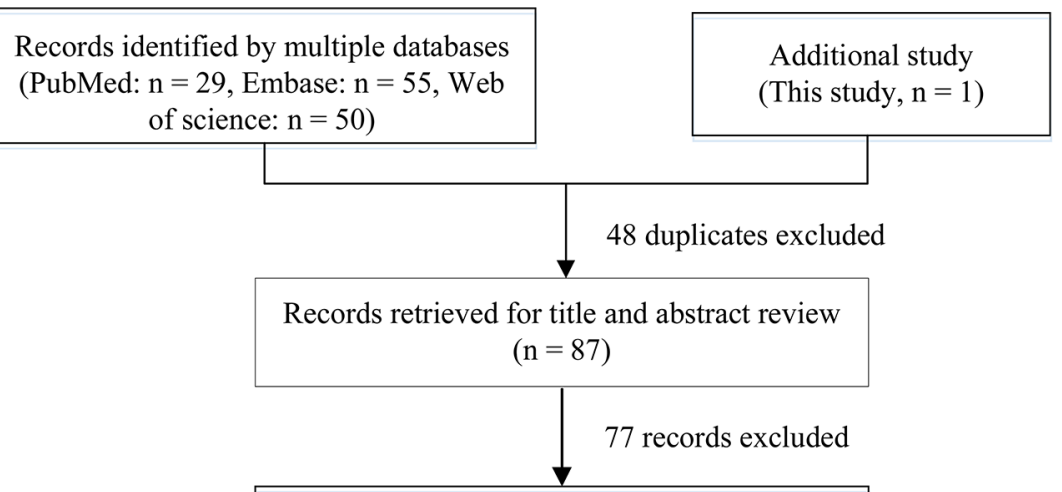

Full-text articles for eligibility evaluation $(\mathrm{n}=10)$

Eligible studies included to assess the association between $M B O A T 7$ rs641738 and $\mathrm{HCC}$ risk $(\mathrm{n}=4)$

Full-text articles excluded $(n=6)$ :

- Studies with overlapped data $(n=3)$

- Deviated from HWE $(n=1)$

- Insufficient data to estimate effect size $(n=1)$

- Imputed genotype data $(\mathrm{n}=1)$

FIGURE 1 | Flowchart of literature search and study selection. 
this variant was also unrelated to persistent infection or the clearance of HBV (all $P>0.30$ ).

\section{Meta-Analysis of the Association Between MBOAT7-TMC4 rs641738 and HCC Risk}

We also conducted a systematic review and meta-analysis to further examine the results. Initially, we obtained 134 records by searching PubMed, Embase, and Web of Science databases, and excluded 48 duplicates and 77 records by title and abstract review. After reviewing the title and abstract and assessing the full-text articles, we excluded six studies. The study by Donati (2017) included two cohorts, the Italian non-alcoholic fatty liver disease (NAFLD) cohort and the UK NAFLD cohort (11). In the Italian NAFLD cohort: the rs641738 variant deviated from the HWE in controls $(P=0.031)$. In the UK NAFLD cohort, the number of HCC cases was too small ( $\mathrm{n}=$ $20)$ to meet our inclusion criteria $(n>30)$. Therefore, we excluded this study from our meta-analysis. In addition, the study of Kawaguchi (2018) provided data on NAFLD type 4 and NASHHCC rather than HCC data, therefore, we could not extract sufficient data to estimate the association between rs641738 and HCC risk and excluded this study (19). The remaining four studies were excluded because they had imputed genotype data $(n=1)(20)$ or overlapped data $(n=3)(21-23)$. Finally, we included three eligible articles (8-10) and this study for the meta-analysis (Figure 1).

A total of 2,135 HCC cases and 4,388 controls were included in the meta-analysis. Characteristics are detailed in Table 3. Except for the study by Raksayot (2019) (NOS score =6) (10), all the studies were of high quality (NOS score $\geq 7$ ). First, we performed a meta-analysis using the allelic model (Figure 2). The pooled results demonstrated that MBOAT7-TMC4 rs641738 was not associated with HCC risk ( $\mathrm{OR}=1.10,95 \% \mathrm{CI}=0.99$ $\left.1.23, P_{\text {hetergeneity }}=0.453, I^{2}=0 \%\right)$, which was stable suggested by the sensitivity analysis (Supplementary Figure S2). Egger's or Begg's test did not suggest any publication bias $\left(P_{\text {Egger's test }}=\right.$ $\left.0.723, P_{\text {Begg's test }}=1.000\right)$. Considering that marginally significant results were observed in our study, we performed meta-analyses using other common genetic models. As shown in Table 4, the pooled results in all these models also consistently showed that there was no association between MBOAT7-TMC4 rs641738 and HCC risk. All pooled results were stable (Supplementary Figure S2) and showed no publication bias (Table 4).

\section{DISCUSSION}

We conducted a case-control study including 799 HCC cases and 1412 controls to investigate the effect of MBOAT7-TMC4 rs641738 on HCC risk. Although this variant showed a marginally significant association in TT vs. CC $(P=0.037)$ and recessive model $(P=0.044)$, all other genetic models demonstrated non-significant results. We also performed a systematic review and meta-analysis to explore the role of MBOAT7-TMC4 rs641738 in HCC susceptibility. A total of 2135 HCC cases and 4388 controls were included in the meta-analysis. The results consistently suggested that MBOAT7-TMC4 rs641738 was not associated with HCC risk, even in both the TT vs. CC and recessive models. In addition, our study revealed that this variant was also

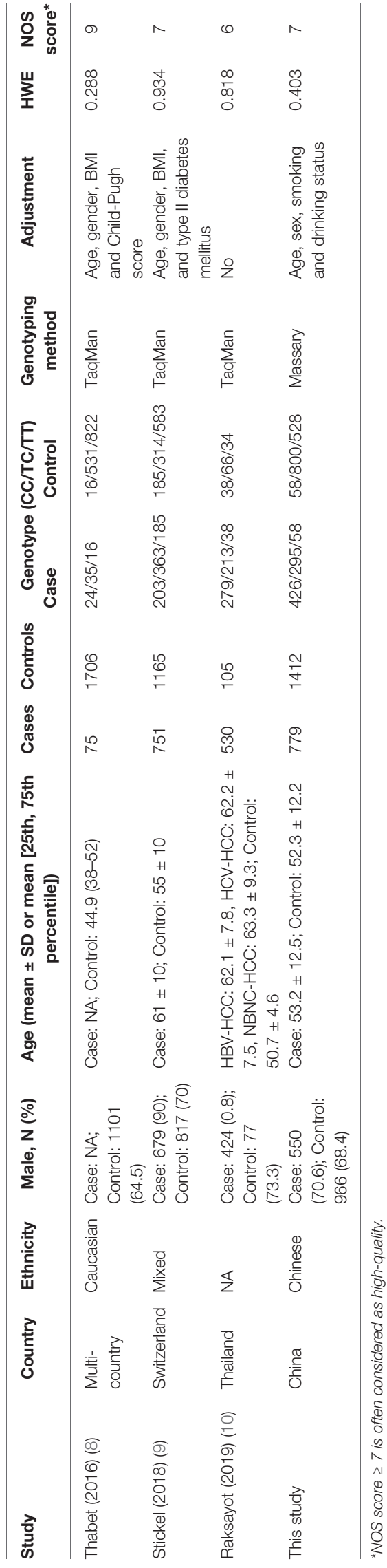


$\begin{array}{ll}\text { Study } & \text { OR }(95 \% \mathrm{CI})\end{array}$

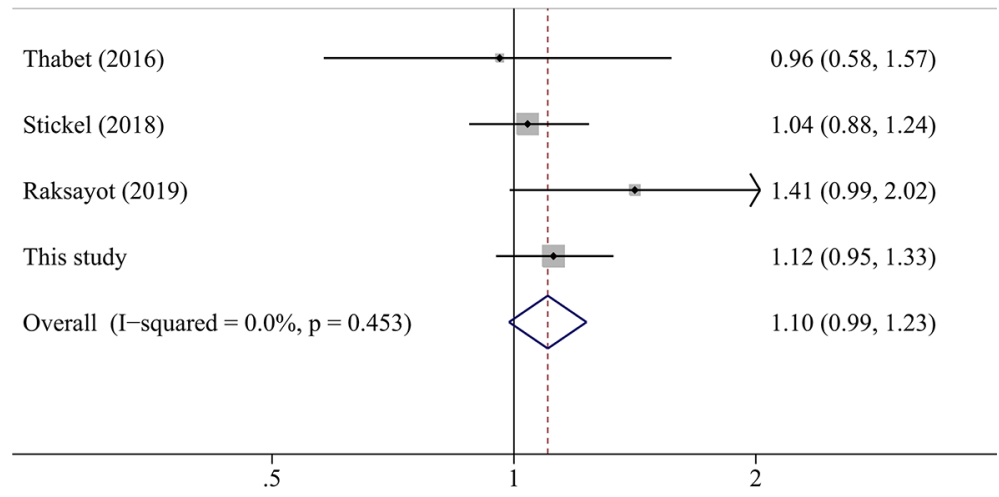

FIGURE 2 | The forest plot of the association between MBOAT7-TMC4 rs641738 and HCC risk under the allelic model.

TABLE 4 | Meta-analysis of the association between MBOAT7-TMC4 rs641738 and HCC risk under other genetic models.

\begin{tabular}{|c|c|c|c|c|c|c|}
\hline Genetic model & OR $(95 \% \mathrm{Cl})$ & $\boldsymbol{P}_{\text {hetergeneity }}$ & $I^{2}(\%)$ & Sensitivity analysis & $P_{\text {Egger's test }}$ & 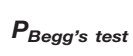 \\
\hline Codominant model (TT vs. CC) & $1.18(0.97-1.43)$ & 0.472 & 0 & Stable & 0.548 & 0.734 \\
\hline Recessive model & $1.17(0.98-1.38)$ & 0.623 & 0 & Stable & 0.529 & 0.734 \\
\hline Additive model & $1.08(0.99-1.18)$ & 0.367 & 5.1 & Stable* & 0.522 & 0.308 \\
\hline
\end{tabular}

*Under the additive model, all pooled results were quite stable, except for excluding the study by Stickel (2018) (9). The pooled OR and 95\% Cl was 1.13 (1.00-1.28) when we excluded the study of Stickel (2018) (9) under the additive model.

not related to persistent $\mathrm{HBV}$ infection in any of the genetic models. To the best of our knowledge, it is the first study to investigate the association between MBOAT7-TMC4 rs641738 and the risk of HCC and persistent HBV infection in Asians.

The rs641738 variant was first identified as a risk locus for alcohol-related cirrhosis (5) and was reported to be associated with nonalcoholic fatty liver disease (NAFLD)/nonalcoholic steatohepatitis (NASH) (24) and liver fibrosis (8). In 2016, Thabet et al. first investigated the association of MBOAT7-TMC4 rs641738 with HCC risk, but did not find a significant result (8). Subsequently, Donati contradicted the conclusion and showed that this variant was predisposed to HCC in NAFLD subjects (11). However, a significant association was not confirmed in later studies (8-10). Even in the NAFLD cohort, MBOAT7-TMC4 rs641738 did not show any association with the risk of Matteoni type 4 or NASHHCC (19). Given the small sample size of the previous studies, we performed this case-control and meta-analysis and confirmed that MBOAT7-TMC4 rs641738 was not associated with HCC risk.

Persistent HBV infection is a crucial risk factor for HCC in China. However, studies on the effect of MBOAT7-TMC4 rs641738 on HBV infection are limited. Here, we also explored the association between MBOAT7-TMC4 rs641738 and persistent $\mathrm{HBV}$ infection. The results exhibited that this variant was not related to spontaneous clearance of $\mathrm{HBV}$, which concurred with a previous report from a Moroccan cohort (25). Interestingly, Thabet et al. reported that MBOAT7-TMC4 rs641738 influenced hepatic inflammation and fibrosis in patients with persistent HBV infection (26). A possible explanation may be that the effect of this variant on liver disease is not associated with HBV or not a causal variant.

The role of MBOAT7-TMC4 rs641738 in liver diseases is still conflicting. MBOAT7 encodes a lysophosphatidylinositol acyltransferase involved in phospholipid metabolism. Some studies have shown that rs641738 reduces mRNA and hepatic MBOAT7 expression, whereas other studies have refuted this (11, 26, 27). Similar contradictions relating to fibrosis and NAFLD have also been reported (25-27). Whether and how MBOAT7TMC4 rs641738 influences liver diseases are still questionable. Despite this, our case-control study and meta-analysis suggest that this variant is not associated with the risk of HCC.

There are some limitations in our study. First, this was a hospital-based case-control study, which may have been influenced by selection bias. However, our multivariate analysis, power calculation, and subsequent meta-analysis ensured the accuracy of our conclusions. Second, our meta-analysis only included four studies because of the limited number of relevant studies. As such, we did not perform further stratified analyses according to factors including age, sex, smoking, or other lifestyle habits. Even so, the results of the meta-analysis are reliable because of robustness, no publication bias, and low heterogeneity. At last, some factors such as HBV viral load are also associated with HCC development, but this study did not collect the information and we failed to make further evaluation based on these factors. Future studies are needed to verify our results. 
In summary, our case-control study and meta-analysis highlight that MBOAT7-TMC4 rs641738 is not associated with HCC risk. Our work also suggests no relationship of this variant with persistent HBV infection in a Chinese population. Further studies are required to validate our results.

\section{DATA AVAILABILITY STATEMENT}

The original contributions presented in the study are included in the article and Supplementary Material, further inquiries can be directed to the corresponding author.

\section{ETHICS STATEMENT}

The studies involving human participants were reviewed and approved by the institutional ethics committee of Tongji Hospital, Tongji Medical College of HUST. The patients/ participants provided their written informed consent to participate in this study. Written informed consent was obtained from the individual(s) for the publication of any potentially identifiable images or data included in this article.

\section{REFERENCES}

1. Siegel RL, Miller KD, Jemal A. Cancer Statistics, 2019. CA: Cancer J Clin (2019) 69(1):7-34. doi: 10.3322/caac.21551

2. Chen W, Zheng R, Baade PD, Zhang S, Zeng H, Bray F, et al. Cancer Statistics in China, 2015. CA: Cancer J Clin (2016) 66(2):115-32. doi: 10.3322/caac.21338

3. Sagnelli E, Macera M, Russo A, Coppola N, Sagnelli C. Epidemiological and Etiological Variations in Hepatocellular Carcinoma. Infection (2019) 48(1):717. doi: 10.1007/s15010-019-01345-y

4. Khemlina G, Ikeda S, Kurzrock R. The Biology of Hepatocellular Carcinoma: Implications for Genomic and Immune Therapies. Mol Cancer (2017) 16 (1):149. doi: 10.1186/s12943-017-0712-x

5. Buch S, Stickel F, Trepo E, Way M, Herrmann A, Nischalke HD, et al. A Genome-Wide Association Study Confirms PNPLA3 and Identifies TM6SF2 and MBOAT7 as Risk Loci for Alcohol-Related Cirrhosis. Nat Genet (2015) 47(12):1443-8. doi: 10.1038/ng.3417

6. Machado MV, Diehl AM. Pathogenesis of Nonalcoholic Steatohepatitis. Gastroenterology (2016) 150(8):1769-77. doi: 10.1053/j.gastro.2016.02.066

7. Helsley RN, Varadharajan V, Brown AL, Gromovsky AD, Schugar RC, Ramachandiran I, et al. Obesity-Linked Suppression of Membrane-Bound O-Acyltransferase 7 (MBOAT7) Drives Non-Alcoholic Fatty Liver Disease. eLife (2019) 8:1-36. doi: 10.7554/eLife.49882

8. Thabet K, Asimakopoulos A, Shojaei M, Romero-Gomez M, Mangia A, Irving WL, et al. Mboat7 rs641738 Increases Risk of Liver Inflammation and Transition to Fibrosis in Chronic Hepatitis C. Nat Commun (2016) 7:12757. doi: 10.1038/ncomms 12757

9. Stickel F, Buch S, Nischalke HD, Weiss KH, Gotthardt D, Fischer J, et al. Genetic Variants in PNPLA3 and TM6SF2 Predispose to the Development of Hepatocellular Carcinoma in Individuals With Alcohol-Related Cirrhosis. Am J Gastroenterol (2018) 113(10):1475-83. doi: 10.1038/s41395-018-0041-8

10. Raksayot M, Chuaypen N, Khlaiphuengsin A, Pinjaroen N, Treeprasertsuk S, Poovorawan $\mathrm{Y}$, et al. Independent and Additive Effects of PNPLA3 and TM6SF2 Polymorphisms on the Development of Non-B, Non-C Hepatocellular Carcinoma. J Gastroenterol (2019) 54(5):427-36. doi: 10.1007/s00535-018-01533-x

11. Donati B, Dongiovanni P, Romeo S, Meroni M, McCain M, Miele L, et al. Mboat7 rs641738 Variant and Hepatocellular Carcinoma in Non-Cirrhotic Individuals. Sci Rep (2017) 7(1):4492. doi: 10.1038/s41598-017-04991-0

\section{AUTHOR CONTRIBUTIONS}

Conceptualization: NS. Methodology and data curation: PW and YL. Formal analysis: LL and RZ. Writing-original draft preparation: PW. Writing-review and editing: NS and RZ. All authors contributed to the article and approved the submitted version.

\section{FUNDING}

This work was supported by National Natural Science Foundation of China (NSFC-81601839 to NS and NSFC81602407 to LL).

\section{SUPPLEMENTARY MATERIAL}

The Supplementary Material for this article can be found online at: https://www.frontiersin.org/articles/10.3389/fonc.2021. 639438/full\#supplementary-material

12. Chen X, Wang Y, Chen X, Cheng K, Li J, Lou J, et al. Genetic Variants in the Regulatory Region of SLC10A1 Are Not Associated With the Risk of Hepatitis B Virus Infection and Clearance. Infect Genet Evol (2016) 44:495-500. doi: 10.1016/j.meegid.2016.07.043

13. Shen N, Li L, Xu W, Tian J, Yang Y, Zhu Y, et al. A Missense Variant in PTPN12 Associated With the Risk of Colorectal Cancer by Modifying Ras/ MEK/ERK Signaling. Cancer Epidemiol (2019) 59:109-14. doi: 10.1016/ j.canep.2019.01.013

14. Lubin JH, Gail MH. On Power and Sample Size for Studying Features of the Relative Odds of Disease. Am J Epidemiol (1990) 131(3):552-66. doi: 10.1093/ oxfordjournals.aje.a115530

15. Moher D, Liberati A, Tetzlaff J, Altman DG. Preferred Reporting Items for Systematic Reviews and Meta-Analyses: The PRISMA Statement. PloS Med (2009) 6(7):e1000097. doi: 10.1371/journal.pmed.1000097

16. Stang A. Critical Evaluation of the Newcastle-Ottawa Scale for the Assessment of the Quality of Nonrandomized Studies in Meta-Analyses. Eur J Epidemiol (2010) 25(9):603-5. doi: 10.1007/s10654-010-9491-z

17. Begg CB, Mazumdar M. Operating Characteristics of a Rank Correlation Test for Publication Bias. Biometrics (1994) 50(4):1088-101. doi: 10.2307/2533446

18. Egger M, Davey Smith G, Schneider M, Minder C. Bias in Meta-Analysis Detected by a Simple, Graphical Test. BMJ (1997) 315(7109):629-34. doi: 10.1136/bmj.315.7109.629

19. Kawaguchi T, Shima T, Mizuno M, Mitsumoto Y, Umemura A, Kanbara Y, et al. Risk Estimation Model for Nonalcoholic Fatty Liver Disease in the Japanese Using Multiple Genetic Markers. PloS One (2018) 13(1):e0185490. doi: 10.1371/journal.pone.0185490

20. Bianco C, Jamialahmadi O, Pelusi S, Baselli G, Dongiovanni P, Zanoni I, et al. Non-Invasive Stratification of Hepatocellular Carcinoma Risk in NonAlcoholic Fatty Liver Using Polygenic Risk Scores. J Hepatol (2020) 74 (4):775-82. doi: 10.1016/j.jhep.2020.11.024

21. Donati B, Dongiovanni P, Miele L, Rosso C, Maier S, Petta S, et al. MBOAT7 Locus rs641738 Variant Predisposes to Hepatocellular Carcinoma in Nonalcoholic Fatty Liver. Digest Liver Dis (2016) 48(SUPPL. 1):e7-8. doi: 10.1016/j.dld.2015.12.029

22. Stickel F, Buch S, Janet F, Rosendahl J, Morgan MY, Nischalke HD, et al. Genetic Variation of PNPLA3 and TM6SF2 Associate With Hepatocellular Carcinoma in Patients With Alcohol-Related Cirrhosis. J Hepatol (2017) 66(1 Supplement 1):S174. doi: 10.1016/S0168-8278(17)30630-X 
23. Stickel F, Buch S, Rosendahl J, Nischalke H-D, Lammert F, Casper M, et al. Owe-016 Genetic Variants in PNPLA3 and TM6SF2 Predispose to Hepatocellular Carcinoma in Patients With Alcohol-Related Cirrhosis. Gut (2018) 67(Suppl_1):A106. doi: 10.1136/gutjnl-2018-BSGAbstracts.209

24. Mancina RM, Dongiovanni P, Petta S, Pingitore P, Meroni M, Rametta R, et al. The MBOAT7-TMC4 Variant Rs641738 Increases Risk of Nonalcoholic Fatty Liver Disease in Individuals of European Descent. Gastroenterology (2016) 150(5):1219-30.e6. doi: 10.1053/j.gastro.2016.01.032

25. Ezzikouri S, Elfihry R, Chihab H, Elmessaoudi-Idrissi M, Zaidane I, Jadid FZ, et al. Effect of MBOAT7 Variant on Hepatitis B and C Infections in Moroccan Patients. Sci Rep (2018) 8(1):12247. doi: 10.1038/s41598-018-30824-9

26. Thabet K, Chan HLY, Petta S, Mangia A, Berg T, Boonstra A, et al. The Membrane-Bound O-Acyltransferase Domain-Containing 7 Variant rs641738 Increases Inflammation and Fibrosis in Chronic Hepatitis B. Hepatol (Baltimore Md) (2017) 65(6):1840-50. doi: 10.1002/hep.29064
27. Sookoian S, Flichman D, Garaycoechea ME, Gazzi C, Martino JS, Castano GO, et al. Lack of Evidence Supporting a Role of TMC4-rs641738 Missense VariantMBOAT7- Intergenic Downstream Variant-in the Susceptibility to Nonalcoholic Fatty Liver Disease. Sci Rep (2018) 8(1):5097. doi: 10.1038/s41598-018-23453-9

Conflict of Interest: The authors declare that the research was conducted in the absence of any commercial or financial relationships that could be construed as a potential conflict of interest.

Copyright (c) 2021 Wang, Li, Li, Zhong and Shen. This is an open-access article distributed under the terms of the Creative Commons Attribution License (CC BY). The use, distribution or reproduction in other forums is permitted, provided the original author(s) and the copyright owner(s) are credited and that the original publication in this journal is cited, in accordance with accepted academic practice. No use, distribution or reproduction is permitted which does not comply with these terms. 